\title{
Treatment Algorithm for Metastatic Renal Cell Carcinoma - Recommendations Based on Evidence and Clinical Practice
}

\author{
Lothar Bergmann $^{\mathrm{a}} \quad$ Jochaim Beck $^{\mathrm{b}}$ Kathrin Bothe ${ }^{\mathrm{c}}$ Olaf A. Brinkmann ${ }^{\mathrm{d}}$ \\ Stephan Buse $^{\mathrm{e}}$ Peter J. Goebell ${ }^{\mathrm{f}}$ Viktor Grünwald ${ }^{g} \quad$ Konstantin Holzapfel $^{\mathrm{h}}$ \\ Hubert Kübleri Norbert W. Marschner ${ }^{j}$ Gerald Mickischk ${ }^{k}$ Wolfgang Schultze-Seemann' \\ Michael Siebels $^{m} \quad$ Stephan Siemer $^{n} \quad$ Stephan Störkel $^{\circ} \quad$ Jürgen E. Gschwend ${ }^{p}$
}

${ }^{a}$ Medizinische Klinik II, Universitätsklinik Frankfurt; ${ }^{\circ}$ III. Medizinische Klinik und Poliklinik, Universitätsmedizin der Johannes Gutenberg-

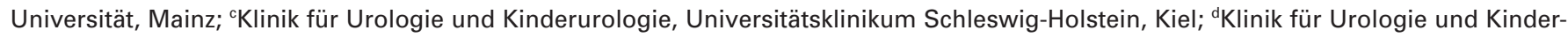

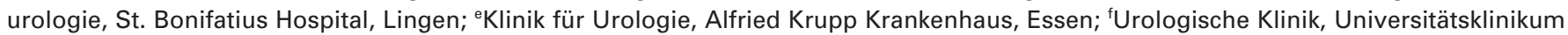

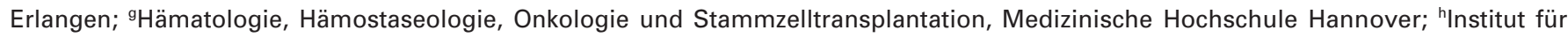
Röntgendiagnostik; 'Urologische Klinik und Poliklinik, Klinikum rechts der Isar, Munich; 'Praxis für Interdisziplinäre Onkologie and Hämatologie, Freiburg i.Br.; ${ }^{k}$ Centrum für Operative Urologie Bremen, Bremen; 'Urologie, Universitätsklinikum Freiburg, Freiburg i.Br.; ${ }^{\mathrm{M}} U$ rologie Pasing, Munich; "Klinik für Urologie und Kinderurologie, Universitätsklinikum des Saarlandes, Homburg; Institut für Pathologie, HELIOS

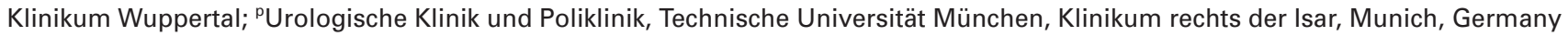

\section{Keywords}

Renal cell cancer $\cdot \mathrm{mRCC} \cdot \mathrm{TKI} \cdot \mathrm{mTOR} \cdot \mathrm{VEGF}$.

Therapy sequences

\section{Summary}

Until a few years ago, the treatment options for metastatic renal cell cancer $(\mathrm{mRCC})$ were very limited. The growing understanding of the molecular pathomechanisms underlying RCC allowed the development of new treatment approaches. Meanwhile, several approved target-oriented substances from different drug classes are available for mRCC. The mechanism of action of vascular endothelial growth factor (VEGF) and VEGF receptor or mTOR inhibition is well documented by phase III trials and reflected in the current guidelines. However, no predictive biomarkers have been identified in $\mathrm{mRCC}$ so far to demonstrate a benefit by a specific compound in an individual patient. Meanwhile, the sequential use of 'targeted therapies' in mRCC has been established as standard treatment. The optimal sequence of available agents is still unclear. A German RCC expert panel discussed and developed an algorithm for the choices of first- and second-line treatment in $\mathrm{MRCC}$ based on established clinical criteria.

\section{Introduction}

Until a few years ago, the treatment options for metastatic renal cell cancer (mRCC) were very limited. The growing understanding of the molecular pathomechanisms underlying RCC allowed the development of new treatment approaches. Today, several approved target-oriented substances from different drug classes are available for mRCC, and other new agents are under clinical development. Meanwhile, the sequential use of 'targeted therapies' in mRCC has been established as standard treatment. The optimal sequence of available agents, however, remains unclear as well as the question of when and with which agent therapy should be initiated.

\section{First-Line Therapy with Target-Oriented Substances}

Interferon (IFN) and interleukin 2 were the first substances approved for RCC. Immunotherapy with these cytokines was the standard for advanced RCC for about 2 decades. With the tyrosine kinase inhibitor (TKI) sunitinib, which blocks particularly the tyrosine kinases of the vascular endothelial growth factor (VEGF) receptors, the first TKI was approved in 2006 for first-line therapy of $\mathrm{mRCC}$ based on positive phase III data [1]. In this phase III study, sunitinib doubled progressionfree survival (PFS) compared to IFN (11 vs. 5 months). With pazopanib, a second TKI is now available in this situation, which was investigated in a phase III study in untreated pa-

\section{KARGER \\ Fax +49 7614520714 \\ Information@Karger.com}

www.karger.com (c) 2014 S. Karger GmbH, Freiburg

2296-5270/14/0373-0136\$39.50/0

Accessible online at:

www.karger.com/ort
Prof. Dr. med. L. Bergmann

Medizinische Klinik II

Universitätsklinik Frankfurt

Theodor-Stern-Kai 7

60590 Frankfurt am Main, Germany

L.Bergmann@em.uni-frankfurt.de 
Table 1. Overview of effectiveness data from the randomized phase II and III studies on sunitinib, pazopanib and bevacizumab/IFN

\begin{tabular}{|c|c|c|c|}
\hline Study [ref.] & Regimen & $\begin{array}{l}\text { Median PFS, } \\
\text { months }\end{array}$ & $\begin{array}{l}\text { Response, } \\
\%\end{array}$ \\
\hline \multirow[t]{2}{*}{ Phase III [1] } & sunitinib & 11 & 31 \\
\hline & IFN & 5 & 6 \\
\hline \multirow[t]{2}{*}{ EFFECT [25] } & sunitinib $4 / 2$ & 8.5 (estimated) & 32 \\
\hline & sunitinib continuous & 7.0 (estimated) & 28 \\
\hline \multirow[t]{2}{*}{ Phase III [2] } & pazopanib & 9.2 & 30 \\
\hline & placebo & 4.2 & 3 \\
\hline \multirow{2}{*}{ AVOREN [3] } & $\mathrm{Bev}+\mathrm{IFN}$ & 10.2 & 31 \\
\hline & placebo + IFN & 5.4 & 13 \\
\hline \multirow[t]{2}{*}{ CALGB [26] } & $\mathrm{Bev}+\mathrm{IFN}$ & 8.5 & 26 \\
\hline & IFN & 5.2 & 13 \\
\hline \multirow[t]{3}{*}{ TORAVA [6] } & sunitinib & 8.2 & 24 \\
\hline & $\mathrm{Bev}+$ tem & 8.2 & 27 \\
\hline & $\mathrm{Bev}+\mathrm{IFN}$ & 16.8 & 39 \\
\hline
\end{tabular}

PFS = Progression-free survival; IFN = interferon;

$\mathrm{Bev}=$ bevacizumab; tem $=$ temsirolimus.

tients or patients previously treated with cytokines versus placebo [2]. In the first-line setting, a significant increase in median PFS from 2.8 months in the placebo arm to 9.2 months was achieved. The monoclonal antibody bevacizumab directed against VEGF is used in combination with IFN for RCC. In the approval-relevant AVOREN study, the combination almost doubled median PFS compared to IFN monotherapy (10.4 vs. 5.5 months) [3]. An overview of the effectiveness data of TKIs and bevacizumab/IFN in first-line therapy is given in table 1. The mTOR inhibitor temsirolimus is approved for first-line therapy of mRCC patients with an unfavorable prognosis. The approval was based on the phase III study Global ARCC in which temsirolimus monotherapy resulted in an improvement of PFS (5.5 vs. 3.1 months) and total survival (10.6 vs. 8.2 months) compared to IFN [4]. In this prognostically unfavorable patient population, however, high-dose IFN (up to 18 million IU $3 \times /$ week) is not to be regarded as a standard and is therefore questionable as a reference.

\section{Targeted Agents for Second-Line Therapy}

Based on a phase III study, the TKI sorafenib is approved for patients with advanced RCC, in whom cytokine therapy has failed or was not indicated [5]. Sorafenib almost doubled PFS in second-line therapy compared to placebo (2.8 vs. 5.5 months). With axitinib, a second TKI has recently become available for the second-line therapy of patients after failure of first-line therapy with sunitinib or a cytokine. In the approval-relevant AXIS study, PFS was increased by 2 months (6.7 vs. 4.7 months) with axitinib compared to sorafenib as reference [6]. Moreover, in second-line therapy, with everolimus an mTOR inhibitor can be used to treat these patients. In the phase III RECORD-1 study, the substance was compared with placebo in patients after failure of at least 1 anti-VEGF therapy, and increased median PFS by 3 months (1.9 vs. 4.9 months) [7]. Because of the mixed patient sample (more than $50 \%$ of the participants had already received 2 previous thera- pies), however, the data supporting the use of everolimus in second-line therapy is limited.

\section{Criteria for Therapy Selection}

In the treatment of advanced RCC, a therapy decision can now be made based on a large number of molecular targeted agents which have shown similar effectiveness in terms of PFS in published studies. Current international guidelines are only of limited value in selecting therapies, as they do not take into consideration the sometimes greatly differing patient profiles, and also because they cannot make statements regarding possible consecutive therapies due to the almost complete absence of evidence-based data on sequential therapy. Furthermore, there are currently no predictive biomarkers for the effectiveness of the targeted agents approved for RCC. Practice-oriented recommendations by accredited experts in RCC therapy are therefore desirable and are increasingly gaining importance. In the absence of molecular predictors, however, the question remains as to which criteria are relevant for the selection of defined targeted agents. To date, only clinical factors have been available for the therapy decision in the first treatment line. An important criterion is histology, since most data on the new substances were collected only or predominantly in clear cell RCC which accounts for $70-80 \%$ of all histological subtypes [8]. The age and comorbidities of the patients and the tolerability of the various substances are also of major importance for therapy selection. Prognostic scores have been developed which include various clinical factors such as hemoglobin, corrected calcium, performance status, time from diagnosis to treatment, and lactate dehydrogenase like that of the Memorial Sloan Kettering Cancer Center (MSKCC), or hemoglobin, corrected calcium, performance status, time from diagnosis to treatment, and neutrophil and platelet count like the Heng score, and which can be used to stratify the patients into 3 risk categories $[9,10]$. The model validation by Kwon et al. [11] on a single-institution evaluation indicated that the Heng model might have a slightly better discriminatory ability than the MSKCC model. More recent is a score devised by the International Kidney Cancer Working Group, which was validated by data from TKItreated patients [12].

A consensus of international experts on therapy selection in $\mathrm{mRCC}$ patients based on clinical factors was published in 2012 by Escudier et al. [13]. The resulting recommendations for sequential therapy for different subtypes of RCC patients are shown in table 2. At an interdisciplinary workshop involving German urologists, oncologists, pathologists and radiologists, a consensus was developed to allow therapy to be optimally tailored to the different patient profiles. The aim was to develop a more individualized therapy recommendation despite the absence of biomarkers. The recommendations presented below are based on the consensus of Escudier et al. 
Table 2. Proposed sequential therapy in different patient subtypes (according to [13])

\begin{tabular}{lll}
\hline Patient type & \multicolumn{2}{l}{ Recommended therapy and therapy situation } \\
\cline { 2 - 3 } & first line & second line \\
\hline 'Indolent' disease & bevacizumab/IFN & sorafenib \\
Aggressive disease & sunitinib & mTOR inhibitor \\
Poor performance status (ECOG 2) & temsirolimus & chemotherapy (e.g. doxorubicin and \\
& & cisplatin-based regimens) \\
\hline
\end{tabular}

[13] with modifications according to the present approval status of additional new agents for mRCC.

The experts were agreed that in addition to the criteria performance status and clinical picture, taking into account the additional criterion 'indolent tumor' allows further characterization of the clinical picture and prognosis with possible consequences for the therapy decision. An 'indolent tumor' means patients who are asymptomatic, with evidence of stable disease or minimal progression on serial imaging.

\section{Characterization of Patient Populations in First-Line Therapy}

With the availability of different molecular principles of action for RCC therapy, affected patients can in principle be treated over the long term by the sequential use of the approved substances. The participants of the interdisciplinary workshop emphasized the value of a simultaneously effective and well tolerated first-line therapy, especially in patients with low tumor dynamics and anticipated long-term therapy, in order to maintain patient acceptance for follow-on therapies and to avoid impairing patients' general condition from the outset by high toxicity that would compromise further therapies. The criteria tumor dynamics, painlessness, symptoms, and prognosis should be used for the selection of a molecular targeted therapy in the first line. They allow differentiation into 4 patient categories.

\section{Group A: Indolent Tumor, Favorable Prognosis}

Group A comprises asymptomatic patients with a favorable prognosis and a small number of metastases restricted to the lungs and/or lymph nodes and who have an 'indolent', i.e. not or only very slowly progressing tumor, controlled by a series of tumor assessments. Patients in this group do not have to be treated immediately in the absence of tumor symptoms and/or rapid progression. Rather, the start of therapy can be delayed until i) accelerated growth of the metastases is observed; or ii) new metastases are detected; or iii) symptoms occur. To allow progression to be identified at an early stage, imaging and clinical investigations should be performed regularly at 2- to 3-monthly intervals. This is important, because in individual cases tumor dynamics can become greatly accelerated, requiring immediate therapeutic intervention. To be differentiated from Group A are asymptomatic patients without progression, who also do not require treatment but close monitoring. They are not listed here as an independent category, as they represent a minority in daily clinical practice.

\section{Group B: Slow but Continuous Tumor Growth,}

\section{Good to Intermediate Prognosis}

Group B comprises patients with an 'indolent' tumor and a favorable to intermediate prognosis, in whom - in contrast to Group A - immediate therapy is indicated because of the more rapid progression. Because of the relatively good prognosis with overall still slow tumor growth, a generally longer therapy period over several therapy lines is to be assumed for these patients. In the experts' opinion, the most important criterion is to achieve a long cumulative PFS in the sequence with simultaneously good tolerability in first-line therapy. In this situation, the choice of a highly effective therapy with a low rate of side effects takes priority to prevent treatment dropout which would be associated with considerable worsening of the patient's prognosis, and potentially also to increase acceptance of follow-on therapies. In this context, the assessment of therapy-related side effects by the patient him-/herself plays a considerable role since this often differs considerably from the physician's assessment. To this day the patient's perspective is neglected too often when recording side effects using the CTCAE (Common Terminology Criteria for Adverse Events).

For RCC patients of Group B, the participants of the interdisciplinary workshop preferably recommend first-line therapy with bevacizumab/IFN. This recommendation corresponds to the treatment proposed by the expert consensus of Escudier et al. [13] who also advocated bevacizumab/IFN for patients with a favorable prognosis and indolent tumor. It is now known that the tolerability of this combined therapy can be markedly improved by reducing the IFN dose: initial evidence from the subgroup analysis of the AVOREN study shows that the rate of IFN-related side effects such as flu-like symptoms, depressiveness, or fatigue can be considerably reduced by administering low-dose IFN (3 million IU $3 \times$ / week) without loss of efficacy. This was prospectively confirmed in the single-arm phase II BEVLiN study $[14,15]$. The median PFS of 15.3 months in this study, which was considered remarkably long by the experts, may indicate that good tolerability of the low-dose therapy regimen could possibly allow longer treatment periods, or may be due to positive selection of patients with a favorable risk profile. According to data of the iOMEDICO-RCC registry from clinical treatment research, the rate of toxicity-related treatment changes of 
Table 3. Overview of recommended therapy algorithm (German Expert Group)

\begin{tabular}{|c|c|c|c|c|c|c|}
\hline Group & Tumor dynamics & Prognosis & Therapy onset & First line & Second line & Third line \\
\hline A & $\begin{array}{l}\text { 'indolent' - } \\
\text { none/slow }\end{array}$ & good & $\begin{array}{l}\text { wait and see, } \\
\text { beginning as for B }\end{array}$ & & & \\
\hline B & $\begin{array}{l}\text { 'indolent' } \\
\text { - intermediate }\end{array}$ & good to intermediate & immediate & $\begin{array}{l}\text { bevacizumab/ } \\
\text { interferon (sunitinib/ } \\
\text { pazopanib) }\end{array}$ & $\begin{array}{l}\text { sunitinib } \\
\text { pazopanib/axitinib }\end{array}$ & everolimus \\
\hline $\mathrm{C}$ & aggressive & $\begin{array}{l}\text { intermediate to } \\
\text { unfavorable }\end{array}$ & immediate & sunitinib/pazopanib & $\begin{array}{l}\text { axitinib } \\
\text { everolimus } \\
\text { sorafenib }\end{array}$ & everolimus/sorafenib \\
\hline $\mathrm{D}$ & aggressive & unfavorable & immediate & $\begin{array}{l}\text { temsirolimus (suni- } \\
\text { tinib/pazopanib) }\end{array}$ & $\begin{array}{l}\text { sunitinib } \\
\text { pazopanib } \\
\text { BSC (if necessary, } \\
\text { sorafenib/everolimus) }\end{array}$ & axitinib \\
\hline
\end{tabular}

approximately $8 \%$ on bevacizumab/IFN occurs much more rarely than during TKI therapy with about $20 \%$ [16].

In patients of Group B, the TKIs sunitinib or pazopanib may be considered alternately as first-line therapy. According to the direct comparative study COMPARZ, comparable efficacy (non-inferiority) of pazopanib and sunitinib was shown in the first therapy line for PFS (8.4 vs. 9.5 months) and response ( 31 vs. $25 \%$ ) with generally better quality of life on pazopanib [17]. The choice of substance should be made on the basis of comorbidities and the expected side effect profile.

Group C: Aggressive Tumor, Intermediate to Poor Prognosis Patients of Group C are to be assigned to the intermediate to unfavorable risk category: they have an aggressive tumor with rapid tumor dynamics. In these cases, an immediate initiation of therapy is indicated, for which the workshop participants assume comparable effectiveness of the approved firstline options in terms of response. Symptomatic patients, however, are assumed to respond more rapidly to TKI therapy than to bevacizumab/IFN therapy. The high response rate (odds ratio) of sunitinib reported from the approval-relevant phase III study (47\%, evaluated by the investigators), however, could not be reproduced in the COMPARZ study. Here, it was $25 \%$ and similar to pazopanib $(31 \%)$ and bevacizumab/ IFN (31\%) [1]. The expert consensus paper of Escudier et al. [13] recommends the administration of sunitinib especially in younger and fit patients of this group, and less for older and/ or comorbid patients. The German workshop participants assessed pazopanib as a now equivalent alternative in this group. Since sorafenib is not generally approved in Europe for first-line therapy due to available data, no recommendation was given on this point [18-21].

\section{Group D: Aggressive Tumor, Unfavorable Prognosis}

Patients of Group D are characterized by an unfavorable prognosis and poor performance status $(\mathrm{ECOG} \geq 2)$. In this case, immediate therapy with temsirolimus is recommended; alternatively, a TKI can be used. In accordance with the inclusion criteria of the global ARCC study, temsirolimus is also recommended for patients with a non-clear cell histology [4].
An overview of the recommended therapy algorithm is shown in table 3 .

\section{Second-Line Therapy}

A long cumulative PFS can be achieved in many patients with advanced RCC by the sequential use of anti-VEGF therapies. Sequential therapies following the first-line therapy should therefore be considered for all patients in whom no severe toxicity problems are to be expected. This applies especially to younger patients and patients without or with only few compromising comorbidities. The experts were basically agreed that an effective and simultaneously well tolerated substance should be chosen for the first-line therapy to motivate patients for follow-on therapies. Currently ongoing studies are expected to provide evidence on optimal sequential therapies. However, before completion of the studies SWITCH I (sunitinib $\rightarrow$ sorafenib vs. sorafenib $\rightarrow$ sunitinib) and SWITCH II (pazopanib $\rightarrow$ sorafenib vs. sorafenib $\rightarrow$ pazopanib) no data are available for the sequential therapy of 2 TKIs. Initial data from the INTORSECT study, in which sunitinib $\rightarrow$ temsirolimus was investigated versus sunitinib $\rightarrow$ sorafenib, suggest that comparable effectiveness levels can be achieved with both sequences [22]. Results of the RECORD III study presented at ASCO 2013, which investigated the therapy sequence everolimus $\rightarrow$ sunitinib vs. sunitinib $\rightarrow$ everolimus, demonstrated that the mTOR inhibitor everolimus is not to be used in the first line [23].

As regards the patient groups defined by the participants of the interdisciplinary workshop, recommendations were expressed for the following therapy sequences:

After first-line therapy with bevacizumab/IFN, patients of Group B should receive TKI therapy (pazopanib, axitinib) in the second and an mTOR inhibitor (everolimus) in the third therapy line (table 3). Sunitinib can also be used. Alternatively, after first-line therapy with a TKI, treatment can be switched in the second line to another TKI with a different receptor profile. There is no absolute cross-resistance between TKIs, as has also recently been shown by the AXIS study [24]. 
It should be considered, however, that the direct sequential use of 2 TKIs may possibly be associated with cumulative gastrointestinal side effects.

In Group C, after first-line therapy with a TKI, a switch can be made to an mTOR inhibitor or a second TKI [24]. When choosing the therapy, particular attention should also be paid to the side effect profile.

If an mTOR inhibitor was used in the first-line therapy for high-risk patients of Group D, in the second-line therapy a switch to a TKI is possible. However, there are no evidencebased data for this sequence.

At the time of switching therapy, it is recommended to take into account the tumor dynamics, as when making the decision at the start of therapy. According to Escudier et al. [13], in the patients with slow progression restraint should be exercised to avoid switching therapy too rapidly; a switch should only be done when progression has been clearly demonstrated, in order to keep other therapy options open as long as possible [11]. The situation should be assessed differently when there is rapid tumor progression for which a rapid switch-over should be considered. In the case of mixed tumor response, for example stabilization in one but progression in another lesion, and when there is evidence of new metastases, isolated progressive metastases should be treated with local surgery and radiotherapy. In the case of relevant progression, however, a switch-over of the systemic therapy is necessary. A therapy change is obligatory if there is unacceptably high toxicity, and if side effect management with symptomatic measures has failed.

\section{Management of Typical Side Effects}

The common side effects of the different targeted agents that severely compromise quality of life include disease- or therapy-associated fatigue with rates of $14-51 \%$. Treatment is difficult. Encouraging and motivating patients to engage in increased physical and sporting activity can be very helpful. Depending on the severity, fatigue can also be improved by reducing the dose of a therapy. The data of the BEVLiN study shows a marked decrease in fatigue without loss of efficacy following reduction of the IFN dose while maintaining the bevacizumab dose [15].

Almost all substances directed against VEGF have a rise in blood pressure as a possible side effect. Blood pressure monitoring before and during therapy and treatment with antihypertensives if hypertension develops are indispensable. A rise in blood pressure on TKI therapy can be regarded as a predictor for a longer PFS, but cannot be utilized pretherapeutically.

Hand-foot syndrome is a typical side effect occurring on TKI therapy. Patients should be informed of this possible side effect before starting the therapy and about preventive measures such as intensive skin and foot care, soft footwear, and pressure relief. The symptomatic treatment of hand-foot syndrome comprises cooling baths and corticoid-containing topical preparations to achieve rapid healing and to allow the therapy to be continued if possible without a dose reduction.

Treatment of the stomatitis and mucositis possibly occurring on TKI, especially on sunitinib and mTOR inhibitors, mainly comprises disinfectant and anesthetic irrigation solutions, and in the case of fungal infections additionally antimycotics.

Many patients treated with a TKI suffer from diarrhea which, however, can usually be controlled with symptomatic measures, and in severe cases by dose reduction. Gastrointestinal toxicity can accumulate in sequential therapy with 2 sequential TKIs, as the data of the AXIS study have shown [24].

Metabolic dysregulations can occur on treatment with mTOR inhibitors or TKIs. They may require treatment but are usually not considered by patients to be burdensome or restrictive. Before starting the therapy and during the further course, blood glucose and lipid values as well as thyroid hormones (thyroid-stimulating hormone) should be monitored regularly and corrected pharmacologically if necessary.

A rare but in individual cases clinically relevant event is the development of non-infectious pneumonitis on everolimus or temsirolimus. This may require interruption of therapy and the use of steroids.

Electrocardiographic abnormalities are also possible during treatment with sunitinib or sorafenib. They are usually asymptomatic, but in serious cases patients should undergo cardiologic evaluation. Patients with a pretherapeutic QT prolongation should not be treated with a TKI.

Particular caution should be exercised with the simultaneous use of medications or other substances (e.g. grapefruit, Saint John's wort) which are metabolized by cytochrome p450, since this can increase or lower the active level of a TKI.

\section{Conclusion}

In advanced RCC, so far no biomarkers are available as predictors for the choice of therapy. Treatment should be chosen on the basis of clinical factors such as prognosis, type of metastases, symptoms, and tumor dynamics, as well as any existing comorbidities. The known toxicity profile should be taken into account when choosing the therapy. Based on the factors mentioned, 4 patient groups are defined and pragmatic therapy options proposed. The therapy decision should not be based solely on the effectiveness of the first-line therapy in terms of PFS and response, but, in the presence of comparable efficacy, should above all take into account tolerability as an important selection criterion. Thanks to the presence of several approved substances, following the failure of a first-line therapy, progressive patients can now be offered follow-on therapies which allow a cumulative increase in PFS. Therefore, the overall therapy sequence should be considered from the 
outset, and the balance between effectiveness and tolerability should be well considered and planned.

\section{Acknowledgement}

We thank all participants of the third interdisciplinary Expert Workshop mRCC (funded by Roche Pharma AG).

\section{Disclosure Statement}

The corresponding author draws attention to the following relationships: J. Beck: advisory (ad) board (Pfizer, Bayer, Astellas), lecture fees
(Roche, Novartis, Bayer). L. Bergmann: ad boards/expert meetings (Astellas, GSK, Novartis, Pfizer, Roche), lecture fees (Novartis, Pfizer, Roche). K. Bothe: ad board (Bayer, Pfizer, GSK, Roche), lecture fees (Pfizer, GSK, Janssen), clinical studies (GSK, Pfizer, Roche). V. Grünwald: ad boards (Astellas, Bayer, GSK, Novartis, Pfizer, Roche), lecture fees (Astellas, Bayer, GSK, Novartis, Pfizer, Roche). P. Goebell: lecture fees, ad boards (Bayer, BristolMyerSquibb, GlaxoSmithKline, Pfizer, Novartis, Roche). J. Gschwend: advisory fees (Astellas, Bayer, Pfizer, Novartis, Janssen, GSK, Roche, Teva). K. Holzapfel: no conflict of interest. H. Kübler: fees and ad boards (Bayer Healthcare), lecture fees (Roche). N. Marschner: ad boards (Pfizer, Bayer, Novartis, Roche), clinical study projects (GSK, Pfizer, Roche, Novartis, Bayer). G. Mickisch: fees and ad boards (Astellas, Bayer Healthcare, GSK, Novartis, Pfizer, Roche). W. Schultze-Seemann: ad board (Amgen) lecture fees (Janssen-Cilag GmbH, Bayer, Roche, Sanofi). M. Siebels: lecture fees (Roche, GSK, Pfizer, Novartis). S. Störkel: study support/ad boards (Merck, Wilex).

\section{References}

1 Motzer RJ, Figlin RA, Hutson TE, et al.: Sunitinib versus interferon alfa in metastatic renal-cell carcinoma. N Engl J Med 2007;356:115-24

2 Sternberg CN, Davis ID, Mardiak J, et al.: Pazopanib in locally advanced or metastatic renal cell carcinoma: results of a randomized phase III trial. J Clin Oncol 2010;28:1061-8.

3 Escudier B, Pluzanska A, Koralewski P, et al. Bevacizumab plus interferon-alfa2a for treatment of metastatic renal cell carcinoma: a randomised, double-blind phase III trial. Lancet 2007;370:210311.

4 Hudes G, Carducci M, Tomczyk P, et al.: Temsirolimus, interferon $\alpha$, or both for advanced renal cell carcinoma. N Engl J Med 2007;336:2271-81.

5 Escudier B, Eisen T Stadler WM, et al.: Sorafenib in advanced clear-cell renal-cell carcinoma. $\mathrm{N}$ Engl J Med 2007;356:125-34.

6 Négrier S, Gravis G, Pérol D, et al.: Temsirolimus and bevacizumab, or sunitinib, or interferon alfa and bevacizumab for patients with advanced renal cell carcinoma (TORACA): a randomised phase II trial. Lancet Oncol 2011;12:673-80.

7 Motzer RJ, Escudier B, Oudard S, et al.: Efficacy of everolimus in advanced renal cell carcinoma: a double-blind, randomised placebo-controlled phase 3 trial. Lancet 2008;372:449-56.

8 Patard JJ. Leray E, Rioux-Leclercq N, et al.: Prognostic value of histologic subtypes in renal cell carcinoma: a multicenter experience. J Clin Oncol 2005;23:2763-71.

$\checkmark 9$ Motzer RJ, Bacik J, Murphy B, et al.: Interferonalfa as a comparative treatment for clinical trials of new therapies against advanced renal cell carcinoma. J Clin Oncol 2002;20:289-96.

10 Heng DY, Xie W, Regan MM, et al.: Prognostic factors for overall survival in patients with metastatic renal cell carcinoma treated with vascular endothelial growth factor-targeted agents: results from a large, multicenter study. J Clin Oncol 2009;27: 5794-9.

11 Kwon WA, Cho IC, Yu A, et al.: Validation of the MSKCC and Heng risk criteria models for predicting survival in patients with metastatic renal cell carcinoma treated with sunitinib. Ann Surg Oncol 2013;20:4397-404.

12 Manola J, Royston P, Elson P, et al.: Prognostic model for survival in patients with metastatic renal cell carcinoma: results from the International Kidney Cancer Working Group. Clin Cancer Res 2011;17:5443-50.

13 Escudier B, Szyczylik C, Porta C, et al.: Treatment selection in metastatic renal cell carcinoma: expert consensus. Nat Rev Clin Oncol 2012;9:327-37.

14 Melichar B, Koralewski P, Ravaud A, et al.: Firstline bevacizumab combined with reduced dose interferon alpha2a is active in patients with metastatic renal cell carcinoma. Ann Oncol 2008;8: 1470-76.

15 Melichar B: Bevacizumab (BEV) + low-dose interferon $\alpha 2 \mathrm{a}$ (IFN) for first-line treatment of metastatic renal cell carcinoma (mRCC): final safety and efficacy data from the prospective BEVLiN study. ESMO 2012;abstr 809P.

16 Auswertung des Tumorregisters Nierenzellkarzinom, Stand September 2012, Registerdatenbank der iOMEDICO AG.

17 Motzer RJ, Hudson TE, Reeves J, et al.: Randomized, open-label, phase III trial of pazopanib versus sunitinib in first-line treatment of patients with metastatic renal-cell carcinoma (mRCC): results of the COMPARZ trial. ESMO 2012; abstr LBA8.

18 Escudier B, Szcylik C, Hutson TE, et al.: Randomized phase II trial of first-line treatment of sorafenib versus interferon alfa-2a in patients with metastatic renal cell carcinoma. J Clin Oncol 2009; 27:1280-9.
9 Jonasch E, Corn P, Pagliaro LC, et al.: Upfront, randomized, phase 2 trial of sorafenib versus sorafenib and low-dose interferon alfa in patients with advanced renal cell carcinoma: clinical and biomarker analysis. Cancer 2010;116:57-65.

20 Procopio G, Verzoni E, Bracarda S, et al.: Sorafenib with interleukin-2 vs. sorafenib alone in metastatic renal cell carcinoma: the ROSORC trial. Br J Cancer 2011;104:1256-61.

21 Rini B, Wilding G, Hudes G, et al.: AMG 386 in combination with sorafenib in patients (pts) with metastatic renal cell cancer (mRCC): a randomized, double-blind, placebo-controlled, phase II trial. J Clin Oncol 2011:29(suppl 7):abstr 309.

22 Hutson T, Escudier B, Esteban E, et al.: Temsirolimus vs sorafenib as second-line therapy in metastatuic renal cell carcinoma: results from the INTORSECT trial. ESMO 2012; abstr LBA22.

23 Motzer JR, Barrios CH, Kim TM, Falcon S, Cosgriff T, et al.: Record-3: phase II randomized trial comparing sequential first-line everolimus (EVE) and second-line sunitinib (SUN) versus first-line SUN and second-line EVE in patients with metastatic renal cell carcinoma (mRCC). J Clin Oncol 2013;Beilage 131.15.

24 Rini BI, Escudier B, Tomczak P, et al.: Comparative effectiveness of axitinib versus sorafenib in advanced renal cell carcinoma (AXIS): a randomised phase 3 trial. Lancet 2011;378:1931-9.

25 Motzer RJ, Hutson TE, Olsen MR, et al.: Randomized phase II multicenter study of the efficacy and safety of sunitinib on the $4 / 2$ versus continuous dosing schedule as first-line therapy of metastatic renal carcinoma: renal EFFECT trial. J Clin Oncol 2011;29(suppl 7):abstr LBA 308.

26 Rini BI, Halabi S, Rosenberg JE, et al.: Phase III trial of bevacizumab plus interferon alfa versus interferon alfa monotherapy in patients with metastatic renal cell carcinoma: final results of CALGB 90206. J Clin Oncol 2010;28:2137-43. 